\title{
LATTICE GAUGE THEORIES AT LARGE $\boldsymbol{n}_{\mathrm{f}}$
}

\author{
Herbert W. HAMBER \\ The Institute for Advanced Study, Princeton, NJ08540, USA
}

Received 29 March 1983

Revised manuscript received 25 April 1983

\begin{abstract}
We investigate the lattice gauge theory with fermions at infinite coupling and show that, after formal integration over the fermion fields, it reduces to the Wilson pure gauge theory in the limit when the number of fermion flavours $n_{\mathrm{f}}$ approaches infinity. When the boson fields are integrated over instead, the action describes a local theory of Fermi fields only. For finite $n_{\mathrm{f}}$ the fermion action contains a finite number of terms.
\end{abstract}

1. Introduction. The idea that fermion fields are the fundamental constituents of matter is not new. Several studies have claimed in the past the equivalence between theories with four-fermion interactions and renormalizable ones [1-3]. The equivalence is in general rather formal and the removal of the cutoff presents often substantial difficulties. Still fourfermion theories have served from the beginning as a useful tool in understanding the dynamics of spontaneous chiral symmetry breaking. On the other hand, it is known that the elimination of the boson fields from a renormalizable gauge theory leads to a nonlocal interaction among the fermions, and not to a simple four-fermion interaction. In recent theories of induced gravity also the gauge field action is obtained as an effective one, after some matter fields representing short distance fluctuations have been integrated out [4-6].

The lattice provides a natural cutoff for investigating these questions in a controlled way, preserving the gauge symmetry of the theory [7]. Recent studies have been able to investigate the question of chiral symmetry breaking on the lattice at strong and intermediate coupling. In this paper we address the question of whether a gauge interaction can be induced on the lattice, when the original theory contains Fermi fields but does not include an explicit kinetic term for the gauge fields. We start out with the Wilson gauge theory [7] with group $\mathrm{SU}(N)$ at in- finite gauge coupling with $n_{\mathrm{f}}$ fermion flavors. After integration over the fermion fields we show that, in the limit when the number of flavors approaches infinity, $n_{\mathrm{f}} \rightarrow \infty$, the Wilson pure gauge action is recovered. In order to determine the equivalence of the theory so defined to a local theory of fermions only, we then perform the functional integral over the gauge boson fields instead. For this task we use known results for the one link integral in $\operatorname{SU}(N)$. Then it can be shown that the interaction involves arbitrary powers of local fermion operators, and the first term in the expansion is a local current-current interaction among the fermions. When the number of fermion flavors is finite the action contains only a finite number of terms, as a consequence of the anticommutation properties of fermion fields.

2. The model. Consider the Wilson lattice gauge theory with fermions defined by the action [7]

$S=S_{\mathrm{G}}+S_{\mathrm{F}}$

with

$$
\begin{aligned}
S_{\mathrm{G}} & =-\frac{1}{g^{2}} \sum_{n, \mu<\nu} \operatorname{tr} U_{n, \mu} U_{n+\mu, \nu} U_{n+\nu, \mu}^{+} U_{n, \nu}^{+}+\text {c.c. } \\
S_{\mathrm{F}} & =k \sum_{f} \sum_{n, \mu}\left[\bar{\psi}_{n+\mu}^{(f)} P_{\mu}^{+} U_{n, \mu}^{+} \psi_{n}^{(f)}+\bar{\psi}_{n}^{(f)} P_{\mu}^{-} U_{n, \mu} \psi_{n+\mu}^{(f)}\right] \\
& -\sum_{f} \sum_{n} \bar{\psi}_{n}^{(f)} \psi_{n}^{(f)}
\end{aligned}
$$


The $U_{n, \mu}$ 's are $N$ by $N$ complex matrices elements of the group $\mathrm{SU}(N)$. The index $f$ labels the different $n_{\mathbf{f}}$ flavors, and we assume that the flavor symmetry is unbroken so that $k_{\mathrm{f}}=k$ for all flavors. $k$ plays the role of a fermion mass parameter in the theory. The matrices $P_{\mu}^{+}$and $P_{\mu}^{-}$are defined as

$P_{\alpha \beta, \mu}^{ \pm}=\frac{1}{2}\left(r \pm \gamma_{\mu}\right)_{\alpha \beta}$,

where $\gamma_{\mu}$ is an euclidean gamma matrix, and $r$ is a parameter that is allowed to vary between 0 and 1 , thus interpolating between Kogut-Susskind and Wilson fermions.

Let us now consider the case of infinite coupling, $1 / g^{2}=0$. Then the action consists only of the fermionic part, and the partition function becomes

$Z_{\mathrm{F}}=\int[\mathrm{d} U][\mathrm{d} \bar{\psi}][\mathrm{d} \psi] \exp \left\{-S_{\mathrm{F}}[U, \bar{\psi}, \psi]\right\}$.

Here $[\mathrm{d} U]$ is the Haar measure for $\operatorname{SU}(N)$, and the fermion integration means

$$
[\mathrm{d} \bar{\psi}][\mathrm{d} \psi]=\prod_{n, \alpha, a, f} \mathrm{~d} \bar{\psi}_{\alpha n}^{a(f)} \prod_{m, \beta, b, f^{\prime}} \mathrm{d} \psi_{\beta m}^{b\left(f^{\prime}\right)} .
$$

Using the Matthews-Salam formula, the integration over the fermion degrees of freedom can be done explicitly, giving the result

$Z_{\mathrm{F}}=\int[\mathrm{d} U][\operatorname{det}(1-k A)]^{n_{\mathrm{f}}}$,

where we have introduced $1-k A$ as the operator defining the fermion quadratic form in (2.2).

It is easy to see that, to lowest order in $k$, the effect of the fermion loops in the full theory is to shift the gauge coupling constant. When the gauge coupling is infinite to start with, the one plaquette interaction term is generated dynamically. To see this expand the statistical factor in (2.5) in powers of $k$. After performing the integrals over the Fermi fields one obtains

$Z_{\mathrm{F}}=R \int[\mathrm{d} U] \exp \left\{-S_{\mathrm{G}}[U]\right\}\left[1+\mathrm{O}\left(n_{\mathrm{f}} k^{6}\right)\right]$.

The constant $R$ is given by

$R=\exp \left\{n_{\mathrm{f}} V 2^{d / 2}\left[\left(\frac{1}{2} k\right)^{2} d\left(r^{2}-1\right)+\left(\frac{1}{2} k\right)^{4} 2 d^{2}\left(r^{2}-1\right)^{2}\right]\right\}$,

with $V$ the volume and $d$ the (even) dimensionality of the lattice. The induced gauge coupling in $S_{\mathrm{G}}$ is given to this order by

$g^{-2}=\left(1+2 r^{2}-r^{4}\right) 2^{d / 2} n_{\mathrm{f}}\left(\frac{1}{2} k\right)^{4}$.

In general the contribution of higher order in $k$ will involve Wilson loops of arbitrary size and shape. But if the limit $n_{\mathrm{f}} \rightarrow \infty$ is taken for fixed $g$ (or $g$ going to zero more slowly than $n_{\mathrm{f}}^{-1 / 2}$ ), then the next contribution is of order $n_{\mathrm{f}} k^{6}$ or $1 / g^{3} n_{\mathrm{f}}^{1 / 2}$ and can therefore be neglected. In this limit one recovers therefore the Wilson pure gauge action

$$
\begin{aligned}
& \lim _{n_{\mathrm{f}} \rightarrow \infty} \int[\mathrm{d} \bar{\psi}][\mathrm{d} \psi] \exp \left\{-S_{\mathrm{F}}[U, \bar{\psi}, \psi]\right\} \\
& \quad=R \exp \left\{-S_{\mathrm{G}}[U]\right\},
\end{aligned}
$$

with the gauge coupling given by eq. (2.9).

So far we have not commented on the question of chiral symmetry and species doubling for the fermion lattice action. Let us briefly recall some important general features of the theory at strong coupling. Chiral symmetry is best discussed in the framework of the Kogut-Susskind fermion action $(r=0)$ [8-10], since in the Wilson case $(r \neq 0)$ chiral symmetry is explicitly broken. In the first formulation one starts out with the fermion action of eq. (2.2) at $r=0$ which is known to describe $16 n_{\mathrm{f}}$ flavors instead of the $n_{\mathrm{f}}$ of the continuum case. In four dimensions the transformation [10]

$\psi_{n}=T_{n} \chi_{n}, \quad T_{n}=\gamma_{1}^{n_{1}} \gamma_{2}^{n_{2}} \gamma_{3}^{n_{3}} \gamma_{4}^{n_{4}}, \quad \bar{\psi}_{n}=\bar{\chi}_{n} T_{n}^{+}$,

turns the action $S_{\mathrm{F}}$ for $r=0$, into

$$
\begin{aligned}
S_{\mathrm{F}} & =\frac{1}{2} \sum_{f} \sum_{n, \mu} \eta_{n, \mu}\left[\bar{\chi}_{n+\mu}^{(f)} U_{n, \mu}^{+} \chi_{n}^{(f)}-\bar{\chi}_{n}^{(f)} U_{n, \mu} \chi_{n+\mu}^{(f)}\right] \\
& -m \sum_{f} \sum_{n} \bar{\chi}_{n}^{(f)} \chi_{n}^{(f)},
\end{aligned}
$$

where $\eta_{n, \mu}=(-1)^{n_{1}+\ldots+n_{\mu-1}}$, and we have rescaled the fermion fields by a factor $\left(\frac{1}{2} k\right)^{1 / 2}$. The mass parameter in this formulation is thus $m=2 / k$.

It is known that, written in this form, the theory describes $4 n_{\mathrm{f}}$ fermion flavors and has a continuous $\mathrm{U}(n) \times \mathrm{U}(n)$ chiral symmetry when the mass parameter $m$ is set to zero. Here $n$ is the number of noncolor indices of the $\chi$ fermion field. The spontaneous breaking of the chiral symmetry was shown in refs. 
[8-10] at strong coupling and large $N$. In this limit the condensate wavefunction was found, in four dimensions, to be [10]

$\left\langle\bar{\psi}_{n} \psi_{n}\right\rangle=N \sqrt{7}$.

The pseudoscalar meson mass can also be computed in this limit, and for small $m$ it is given by

$M_{\mathrm{P}}^{2}=(12 / \sqrt{7}) m+\mathrm{O}\left(m^{2}\right)$.

It is also known that these results remain qualitatively correct for intermediate and weak $g^{2}[11,12]$.

3. The fermion theory. In this section we will derive the purely fermionic theory that corresponds to the action $S_{\mathrm{F}}$ of eq. (2.2). In the previous section we choose to integrate over the Fermi fields in order to show the form of the effective gauge boson action. Here we will do the integral over the link variables instead, keeping the fermions unintegrated. To do the integral over the $U_{n, \mu}$ variables we use known results for the one-link integral. Define the fields $A$ and $\bar{A}$ as

$A_{n, \mu}^{a b}=\bar{\psi}_{n+\mu}^{a} P_{\mu}^{+} \psi_{n}^{b}, \quad \bar{A}_{n, \mu}^{a b}=\bar{\psi}_{n}^{a} P_{\mu}^{-} \psi_{n+\mu}^{b}$,

and we have suppressed spinor and flavor indices for notational simplicity. Next we define the function $W(\bar{A}, A)$ as

$\exp [W(\bar{A}, A)]=\int[\mathrm{d} U] \exp \left[\operatorname{tr}\left(\bar{A} U+U^{+} A\right)\right]$,

and for the induced fermion action we obtain the expression

$$
\begin{aligned}
& \ln \int[\mathrm{d} U] \exp \left\{-S_{\mathrm{F}}[U, \bar{\psi}, \psi]\right\} \\
& =\sum_{n, \mu} w\left(\bar{A}_{n, \mu}, A_{n, \mu}\right)+\sum_{n} \bar{\psi}_{n} \psi_{n} .
\end{aligned}
$$

For the gauge group $\mathrm{U}(1)$ the function $W(\bar{A}, A)$ is just $\ln I_{0}\left(2 \operatorname{tr}(\bar{A} A)^{1 / 2}\right.$, where $I_{0}$ is the modified Bessel function. In the case of the group $\mathrm{SU}(N)$ the function $\exp [W(\bar{A}, A)]$ is known explicitly for $\mathrm{SU}(2), \mathrm{SU}(3)$ and large $N[13-17]$. A few terms in the series expansion of $W$ for small $\operatorname{tr}(\bar{A} A)^{1 / 2}$ ("strong coupling") are known for any $N$

$$
\begin{aligned}
& W(\bar{A}, A)=N^{-1} \operatorname{tr}(\bar{A} A) \\
& \quad+\left[2 N\left(N^{2}-1\right)\right]^{-1}\left[N^{-1}(\operatorname{tr} \bar{A} A)^{2}-\operatorname{tr}(\bar{A} A)^{2}\right]+\ldots \\
& \quad+(2 N)^{-1}(\operatorname{det} A+\operatorname{det} \bar{A})+\ldots
\end{aligned}
$$

For weak coupling one has [13]

$$
W(\bar{A}, A)=2 N \operatorname{tr}(\bar{A} A)^{1 / 2}-\frac{1}{2} \sum_{a b} \ln \left(\lambda_{a}^{1 / 2}+\lambda_{b}^{1 / 2}\right)-\frac{3}{2},
$$

where the $\lambda_{a}$ 's are the eigenvalues of $(\bar{A} A)$. Thus, as in the case of the logarithm of the Bessel function, the function $W(\bar{A}, A)$ has a quadratic behaviour in $(\bar{A} A)^{1 / 2}$ for small argument and becomes linear for large argument. In order to see the explicit expression for $W$ as a function of the fermion fields introduce the composite fields

$M_{\alpha \beta, n}=N^{-1} \bar{\psi}_{\alpha n}^{a} \psi_{\beta n}^{a}$,

and

$$
\begin{aligned}
& B_{\alpha_{1} \ldots \alpha_{N, n}}=(1 / N !) \epsilon_{\alpha_{1} \ldots \alpha_{N}} \psi_{\alpha_{1}, n}^{a_{1}} \ldots \psi_{\alpha_{N}, n}^{a_{N}}, \\
& \bar{B}_{\alpha_{1} \ldots \alpha_{N}, n}=(1 / N !) \epsilon_{a_{1} \ldots a_{N}} \bar{\psi}_{\alpha_{1}, n}^{a_{1}} \ldots \bar{\psi}_{\alpha_{N}, n}^{a_{N}}
\end{aligned}
$$

Then substituting (3.6) and (3.7) in (3.3) we obtain

$$
\begin{aligned}
& \ln \int[\mathrm{d} U] \exp \left\{-S_{\mathrm{F}}[U, \bar{\psi}, \psi]\right\} \\
& \quad=N \sum_{n, \mu}\left\{-\operatorname{tr} M_{n} P_{\mu}^{-} M_{n+\mu} P_{\mu}^{+}+\left[N^{2} / 2\left(N^{2}-1\right)\right]\right. \\
& \quad \times\left[N^{-1}\left(\operatorname{tr} M_{n} P_{\mu}^{-} M_{n+\mu} P_{\mu}^{+}\right)^{2}\right. \\
& \left.\left.\quad-\operatorname{tr}\left(M_{n} P_{\mu}^{-} M_{n+\mu} P_{\mu}^{+}\right)^{2}\right]+\ldots\right\} \\
& \quad+\sum_{n, \mu}\left[\bar{B}_{\alpha_{1} \ldots \alpha_{N}, n} P_{\beta_{1}}^{+\alpha_{1}} \ldots P_{\beta_{N}}^{+\alpha_{N}} B_{\beta_{1} \ldots \beta_{N}, n+\mu}\right. \\
& \left.\quad+\left(+\leftrightarrow-, B_{n+\mu} \rightarrow B_{n}, \bar{B}_{n} \rightarrow \bar{B}_{n+\mu}\right)\right] .
\end{aligned}
$$

Note that for $r=0$ the first term is just a familiar current-current interaction

$-N \sum_{f, f^{\prime}} \sum_{n, \mu}\left(\bar{\psi}_{n}^{a(f)} \gamma_{\mu} \psi_{n+\mu}^{b(f)}\right)\left(\bar{\psi}_{n+\mu}^{b\left(f^{\prime}\right)} \gamma_{\mu} \psi_{n}^{a\left(f^{\prime}\right)}\right)$

For $r \neq 0$, although less familiar, it still involves the conserved vector current on the lattice.

Because of the anticommutation properties of the fermion fields, one can show that for finite $n_{\mathrm{f}}$ the series in $(\bar{A} A)^{1 / 2}$ for $W(\bar{A} A)$ actually truncates. One has the following properties

$\bar{\psi}^{k}=\psi^{k}=0$ for $k>N n$,

and therefore 
$M^{k}=0 \quad$ for $\quad k>N n$

$\bar{B}^{k}=B^{k}=0 \quad$ for $\quad k>n$.

Here $n$ is equal to $n_{\mathrm{f}}$ or $4 n_{\mathrm{f}}$ depending on whether one is considering Wilson or Kogut-Susskind fermions. As the number of fermion flavors increases, the number of nonzero terms in the series for the effective fermion action increases, and involves higher and higher powers of the fermion fields. The effect of these terms will be equivalent to gradually order the $U_{n, \mu}$ fields (rotate them towards the identity in group space, up to a gauge transformation), in complete analogy with the pure gauge case when the coupling constant $g^{2}$ is decreased.

In conclusion let us summarize our results. We have shown that the Wilson lattice gauge theory with $n_{\mathrm{f}}$ fermion flavors at infinite coupling is equivalent to the pure gauge theory when the number of fermion flavors is sent to infinity, with a coupling given by eq. (2.9). We then showed that the same theory can be regarded as describing a set of interacting Fermi fields only, with an action that is given by a finite number of local terms for finite $n_{\mathrm{f}}$.

After this work was completed, I received a preprint by Bander [18], in which similar ideas are developed. He does not consider the case of fermion fields.
I wish to thank Steve Adler for a useful conversation. This research was supported by the US Department of Energy under grant no. DE-AC02-76ER02220.

\section{References}

[1] Y. Nambu and G. Jona-Lasinio, Phys. Rev. 122 (1961) 345 .

[2] W. Kerler, Phys. Lett. 69B (1977) 355 ; and preprint (1981).

[3] T. Eguchi, Phys. Rev. D17 (1978) 611.

[4] A. Zee, Phys. Rev. D23 (1981) 858.

[5] S. Adler, Rev. Mod. Phys. 54 (1982) 729.

[6] D. Amati and G. Veneziano, Phys. Lett. 105B (1981) 358 , and references therein.

[7] K.G. Wilson, Phys. Rev. D10 (1974) 2445; and in: New phenomena in subnuclear physics (Erice, 1975), ed. A. Zichichi (Plenum, New York, 1977).

[8] J.M. Blairon, R. Brout, F. Englert and J. Greensite, Nucl. Phys. B180 [FS2] (1981) 439.

[9] H. Klugberg-Stern, A. Morel, O. Napoly and B. Peterson, Nucl. Phys. B190 [FS3] (1981) 504.

[10] N. Kawamoto and J. Smit, Nucl. Phys. B192 (1982) 100 and ITFA preprint (1981), and references therein.

[11] H. Hamber and G. Parisi, Phys. Rev. Lett. 47 (1981) 1795.

[12] H. Hamber and G. Parisi, Phys. Rev. D27 (1983) 208.

[13] E. Brezin and D. Gross, Phys. Lett. 97B (1980) 120.

[14] R. Brower and M. Nauenberg, Nucl. Phys. B1 80 [FS 2] (1981) 221

[15] I. Bars and F. Green, Phys. Rev. D20 (1979) 3311.

[16] I. Bars, J. Math. Phys. 21 (1980) 2678.

[17] S. Samuel, J. Math. Phys. 21 (1980) 2695.

[18] M. Bander, Phys. Lett. 126B (1983) 463. 